\title{
Radiologic Results of Three-Dimensional Templating for Total Shoulder Arthroplasty
}

\author{
Sung-Hyun Cho, MD, Jinyoung Jeong, MD \\ Department of Orthopedic Surgery, St. Vincent's Hospital, College of Medicine, The Catholic University of Korea, Suwon, Korea
}

Background: The purpose of this study was to evaluate the radiologic results of total shoulder arthroplasty using computerized three-dimensional (3D) templating in preoperative planning.

Methods: Ten patients who underwent total shoulder arthroplasty using 3D templating preoperatively were enrolled in this study. A specialized computer program was used to reconstruct the 3D images of the shoulder from the computed tomographic images. The 3D images of various sizes of prostheses were used as the template in surgical planning of the shoulder arthroplasty. The size of the glenoid, humeral head, and stem measured in 3D templating were compared with those used in actual operation. Anatomical parameters, such as humeral head size, radius of curvature, and greater tuberosity to humeral head distance of the replaced shoulder, were measured and compared with those of the contralateral normal shoulder.

Results: The agreement rates between the glenoid size, head size, head thickness, and stem size estimated preoperatively by 3D templating and those measured in operation were $100 \%, 100 \%, 100 \%$, and $80 \%$, respectively. The difference in humeral head size, radius of curvature, and greater tuberosity to humeral head distance between the replaced shoulder and contralateral shoulder was $1.31 \mathrm{~mm}, 0.87 \mathrm{~mm}$, and $1.17 \mathrm{~mm}$, respectively.

Conclusions: In total shoulder arthroplasty, 3D templating seems to enable accurate prediction of sizes of the prostheses to be inserted and thus replication of normal anatomy.

Keywords: Shoulder arthroplasty, Three-dimensional templating, Radiologic results

Replicating normal anatomy of the shoulder is an important principle in the arthroplasty of the shoulder joint. A change in the thickness of the humeral head by $5 \mathrm{~mm}$ or more could tighten the capsule and cuff and reduce the range of motion of the shoulder by $23^{\circ}$ to $30^{\circ}{ }^{1)}$ Inferior malposition of the humeral head by $4 \mathrm{~mm}$ or more could lead to an increase in subacromial contact, and malposition in any direction by $8 \mathrm{~mm}$ could result in a significant decrease in passive range of motion of the shoulder. ${ }^{2)}$ Also, it is thought that a more anatomic reconstruction will bet-

Received July 3, 2019; Accepted August 13, 2019

Correspondence to: Jinyoung Jeong, MD

Department of Orthopedic Surgery, St. Vincent's Hospital, College of Medicine, The Catholic University of Korea, 93 Jungbu-daero, Paldal-gu,

Suwon 16247, Korea

Tel: +82-31-881-8850, Fax: +82-31-254-7186

E-mail: osjeong@hotmail.com ter facilitate optimal function after total shoulder arthroplasty. $^{3-5)}$

Identifying normal anatomy and pathologic changes is the first step in developing a strategy for replicating normal geometry in shoulder arthroplasty. Accurate measurement of sizes of the prostheses to be inserted is at the basis of this step. It is not easy, however, to preoperatively measure or determine the exact size of the prosthetic stem and head that will fit normal anatomy by using the conventional two-dimensional (2D) templating on plain X-rays. The three-dimensional (3D) images of the native humerus and prostheses may be useful in selecting the proper size for restoring normal anatomy.

Therefore, the hypothesis of this study was that 3D templating would accurately replicate normal anatomy of the proximal humerus in total shoulder arthroplasty. A computerized 3D reconstruction program was used in preoperative planning and radiologic results after the sur- 
Cho et al. Three-Dimensional Templating for Total Shoulder Joint Arthroplasty

Clinics in Orthopedic Surgery • Vol. 12, No. 2, $2020 \bullet$ www.ecios.org

gery were evaluated to test this hypothesis.

\section{METHODS}

This study was conducted in accordance with the ethical guidelines of the Declaration of Helsinki. The protocol was reviewed and approved by the Institutional Review Board of St. Vincent Hospital (IRB No. VC11RISE0226). Informed consent was waived for the retrospective design.

Ten patients who underwent total shoulder arthroplasty using 3D templating preoperatively were enrolled in this study. There were four men and six women with a mean age of 64.6 years (range, 51-81 years). They were followed up for an average of 25.3 months (range, 15-33 months), and plain radiographs of the shoulder of these patients were reviewed. Except one patient with avascular necrosis of the humeral head, all patients $(n=9)$ had primary osteoarthritis. Single experienced surgeon (JJ) did all the operations. A specialized computer program developed for surgical simulation in shoulder arthroplasty (CCF Surgical Planner, Cleveland, OH, USA) was used to reconstruct the 3D images of the shoulder from DICOM files of the computed tomography (CT) images (images of $1 \mathrm{~mm}$ axial cut and $\mathrm{B} 60$ reconstruction kernel). The 3D images of various sizes of prostheses (Global Advantage; DePuy, Warsaw, IN, USA) were included in the computer program and were used as the template in the surgical planning of shoulder arthroplasty (Fig. 1). Proper sizes and positions of the prostheses were selected by applying $3 \mathrm{D}$ images of the prostheses to the reconstructed 3D images of the shoulder. The size of glenoid was considered proper if $100 \%$ of the glenoid prosthesis was supported by underlying subchondral bone and the peg of the glenoid implant was within the glenoid cavity. The humeral head size was defined as the radius of a circle best-fitting the native humeral head or prosthetic head. The humeral head thickness was defined as the longest distance between the head surface and the anatomic neck plane. The tuberosityto-humeral head distance was defined as the distance between the most superior point on the greater tuberosity and the most superior point of the humeral head (Fig. 2). The proper stem size was determined as the largest stem

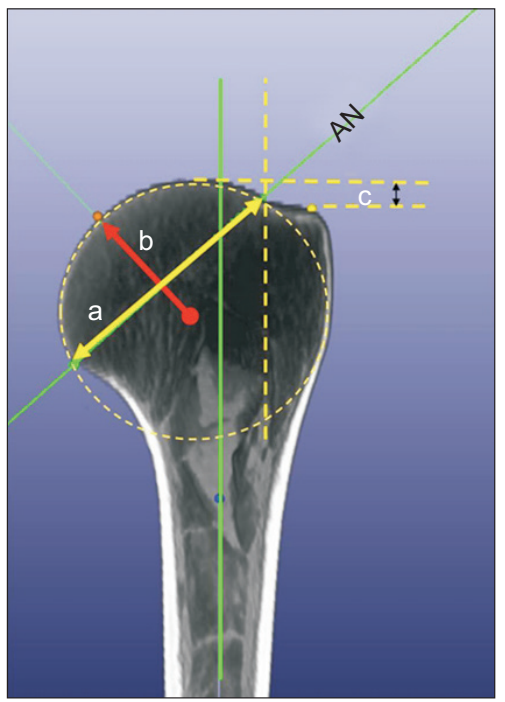

Fig. 2. Anatomical parameters. a: humeral head size, b: radius of curvature, c: greater tuberosity to humeral head distance, AN: anatomical neck.

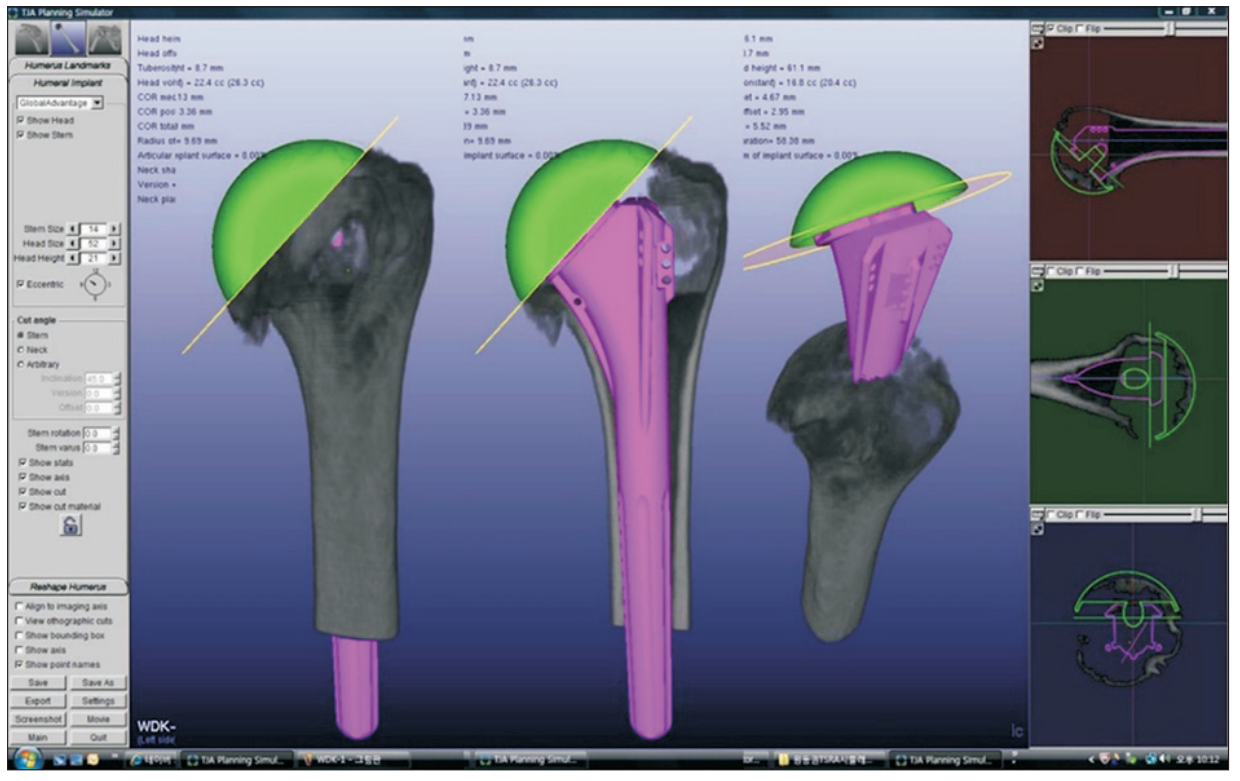

Fig. 1. Computer program for threedimensional (3D) templating. The 3D images of prostheses in various sizes are used as a template in the surgical planning of shoulder arthroplasty. 
Cho et al. Three-Dimensional Templating for Total Shoulder Joint Arthroplasty

Clinics in Orthopedic Surgery • Vol. 12, No. 2, $2020 \bullet$ www.ecios.org

whose distal one-third matched the inner diameter of the corresponding medullary canal. In the surgical field, the largest reamer size that contacts the inner cortex of midshaft of the humerus was considered the right size to be used. The sizes of the glenoid, humeral head, and stem measured on 3D templating were compared with those used in actual operation. Anatomical parameters, such as humeral head size, glenoid component size, radius of curvature, and greater tuberosity to humeral head distance of the replaced shoulder were measured and compared with those of the contralateral normal shoulder in plain radiographs (Fig. 3). We used the Mann-Whitney test to compare these parameters between the normal side and the operated side because of the relatively small sample size.
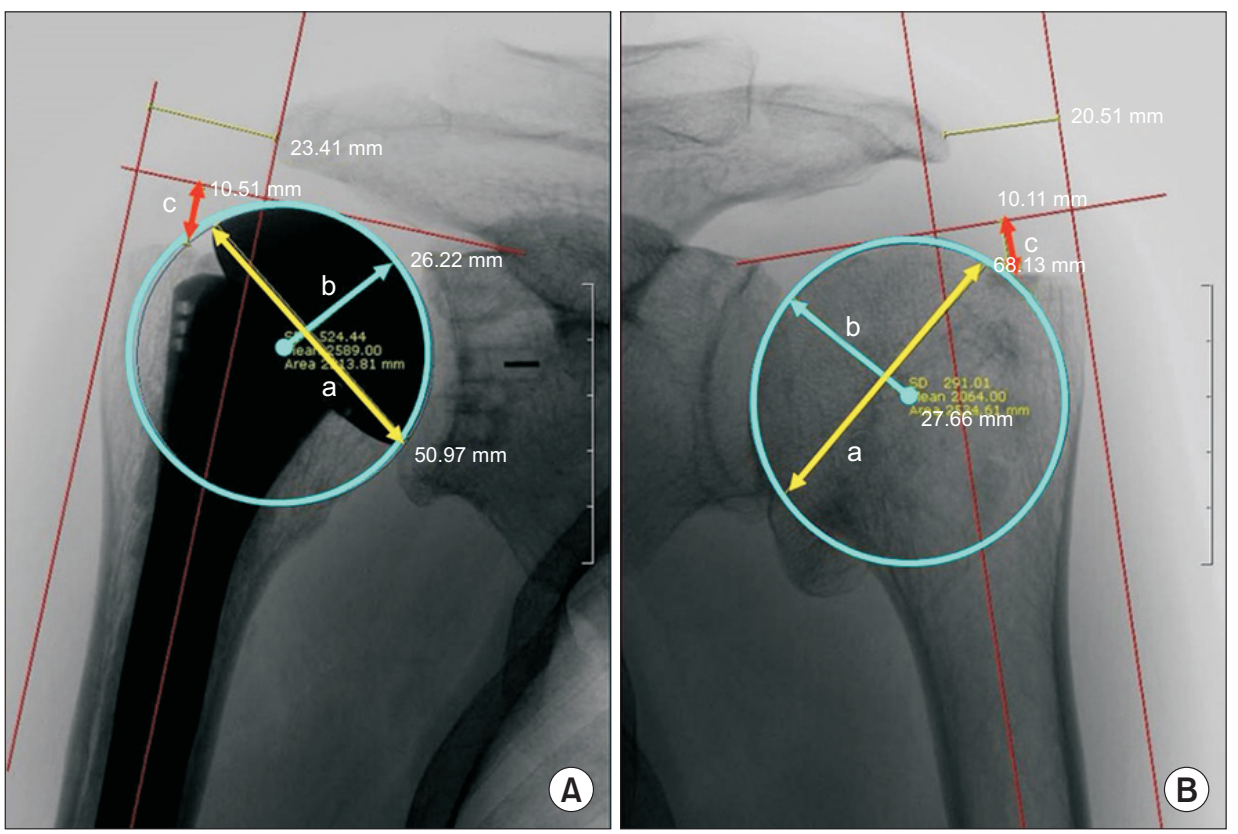

Fig. 3. Anatomical parameters are compared between the replaced shoulder (A) and the contralateral normal shoulder (B) a: humeral head size, b: radius of curvature, c: greater tuberosity to humeral head distance.

Table 1. Three-Dimensional Templating Results and Actual Prosthesis Sizes

\begin{tabular}{|c|c|c|c|c|c|c|c|c|c|c|}
\hline \multirow{2}{*}{ Case } & \multicolumn{5}{|c|}{ Templated size } & \multicolumn{5}{|c|}{ Actual prosthesis size } \\
\hline & Glenoid & $\begin{array}{l}\text { Glenoid } \\
\text { type }\end{array}$ & HH size & $\begin{array}{c}\mathrm{HH} \\
\text { thickness }\end{array}$ & Stem & Glenoid & $\begin{array}{l}\text { Glenoid } \\
\text { type }\end{array}$ & $\mathrm{HH}$ size & $\begin{array}{c}\mathrm{HH} \\
\text { thickness }\end{array}$ & Stem \\
\hline$A$ & 48 & $C$ & 48 & 21 & 14 & 48 & E2 & 48 & 21 & 14 \\
\hline$B$ & 44 & C & 44 & 18 & 10 & 44 & C & 44 & 18 & 10 \\
\hline$C$ & 48 & E5 & 48 & 18 & 14 & 48 & $E$ & 48 & 21 & 14 \\
\hline$D$ & 52 & E5 & 52 & 21 & 12 & 52 & $E$ & 52 & 21 & 12 \\
\hline$E$ & 44 & E11 & 44 & 18 & 12 & 44 & $C$ & 44 & 21 & 12 \\
\hline $\mathrm{F}$ & 48 & E12 & 48 & 18 & 12 & 48 & E10 & 48 & 18 & 12 \\
\hline G & 44 & E10 & 44 & 18 & 12 & 44 & E10.5 & 44 & 18 & 12 \\
\hline$H^{\dagger, \neq}$ & 48 & C & $48(52)$ & 21 & 14 & 48 & C & 48 & 21 & 12 \\
\hline$I^{\dagger, \ddagger}$ & 44 & E2 & 44 (48) & 18 & 12 & 44 & E10 & 44 & 18 & 10 \\
\hline$J^{\dagger}$ & 40 & C & $40(44)$ & 21 & 10 & 40 & C & 40 & 18 & 10 \\
\hline
\end{tabular}

$\mathrm{HH}$ : humeral head.

*Glenoid type: C, centric type glenoid implant; E5, eccentric type glenoid implant with 5 o'clock position (the number indicates the position of the hour hand of a clock). ${ }^{\dagger}$ The templated size of the humeral head was smaller than the ideal size (numbers in parentheses indicate the ideal size of the glenoid implant after three-dimensional templating) because of the small glenoid size. ${ }^{\ddagger}$ The actual stem size was different from the templated size. 
For statistical analysis, R software ver. 3.6.0 (R Foundation, Vienna, Austria) was used with $p$-values less than 0.05 considered to reveal statistically significant difference. The accuracy of measuring details of the humerus by the computer program used in this study has already been proved in previous studies. ${ }^{6,7)}$

\section{RESULTS}

The rate of agreement was high between the 3D templating results and the actual size of implanted prostheses. On the glenoid size, the templated prosthesis matched the implanted prosthesis in $100 \%$ of the cases. With regard to glenoid type, eccentric and centric classification matched in $80 \%$ of the cases, but the detail of arc size was slightly different between the 3D templating results and actual prostheses. Humeral head size also showed 100\% match between templating and intraoperative results. In three cases (H-J), humeral heads with sizes smaller than the initial sizes (parentheses in Table 1) were selected during templating because the humeral head of the prosthesis could not be bigger than the glenoid. This expectation was consistent with intraoperative findings. Also, the humeral head thickness of the implanted prosthesis matched templating results in $70 \%$ of the cases, and the agreement rate was $80 \%$ for the humerus stem size.

The radiologic parameters on X-ray performed after total shoulder arthroplasty showed successful anatomical restoration. The difference between the operated side and contralateral side regarding the humeral head size, radius of curvature, and greater tuberosity to humeral head distance was $1.31 \mathrm{~mm}, 0.87 \mathrm{~mm}$, and $1.17 \mathrm{~mm}$, respectively ( $p$ $=0.65, p=0.94$, and $p=0.65$, respectively) (Table 2).

\section{DISCUSSION}

Anatomical variations in normal shoulders are not rare. ${ }^{8)}$

Table 2. Radiological Difference between the Contralateral Side and Operated Side

\begin{tabular}{lcc}
$\quad$ Variable & Difference $(\mathrm{mm})$ & $p$-value \\
HH size & $1.31 \pm 1.09$ & 0.65 \\
ROC & $0.87 \pm 0.69$ & 0.94 \\
GT-HH & $1.71 \pm 1.43$ & 0.65 \\
\hline
\end{tabular}

Values are presented as mean \pm standard deviation. The average of absolute difference was calculated.

$\mathrm{HH}$ : humeral head, ROC: radius of curvature, GT-HH: greater tuberosity to humeral head distance.
Approximately 20\% of normal shoulders have an excessive valgus or varus neck-shaft angle of the proximal humerus (below $130^{\circ}$ or above $140^{\circ}$ ). ${ }^{9)}$ In other words, one in five patients may have excessive anatomical variations, which needs to be considered in surgical planning. A mismatch between the resected humeral head and the prosthetic head to be implanted is not uncommon in real situation. This is especially true in shoulders with excessive variations because inventories of prostheses are not enough to accommodate all the instances. Therefore, accurate preoperative planning or templating is important for proper size selection of prostheses, and in cases where the proper size is not available, surgical modifications should be made to overcome these mismatches.

In 2D templating-the conventional method used to preoperatively estimate the size of prostheses to be implanted-a transparent plastic plate with figures of various sizes of prostheses is superimposed on radiographs of the shoulder. However, it is not easy to measure 3D structures or to select the best-fit prosthesis by using a $2 \mathrm{D}$ template on $2 \mathrm{D}$ radiographs, which can result in a mismatch between the preoperative and postoperative sizes. ${ }^{10)}$

Also, because the range of prosthesis size is limited, surgeons sometimes have to perform additional bone cutting of the humeral neck or use a larger humeral component than the patient's anatomical size. In the current study, the sizes of the implant predicted preoperatively by using $3 \mathrm{D}$ templating corresponded with the sizes of the implant actually placed in surgery in more than $70 \%$ of the cases. In particular, the prediction was $100 \%$ accurate for the glenoid component. Because the humeral head component should not be bigger than the glenoid component in total shoulder arthroplasty, surgeons occasionally fit the size of humeral component to that of the glenoid implant. This narrows the choice for adequate prostheses particularly among Asian patients because of their small glenoid size. We estimated the size of glenoid prosthesis by using $3 \mathrm{D}$ templating before surgery and achieved $100 \%$ concordance with the actual size. This led to the high agreement rate between the planned size and actual size of the humeral head component. Still, there was size mismatch of the humeral head between the templating results and real prosthesis in $30 \%$ of the cases; we assumed this may be from intraoperative variations such as errors in cutting accuracy and insertion angle of the stem.

The difference in humeral head size, radius of curvature, and greater tuberosity to humeral head distance between the operated side and contralateral side was 1.31 $\mathrm{mm}, 0.87 \mathrm{~mm}$, and $1.17 \mathrm{~mm}$, respectively. There was no statistical significance in these differences, which means 
anatomical restoration was accomplished. Saifi et al. ${ }^{11)}$ compared the 3D templated size and implanted glenoid component size and reported that surgeons tended to select a bigger implant than the templating component. Scalise et al. ${ }^{12)}$ found that $3 \mathrm{D}$ image planning overestimated glenoid component size. In spite of different opinions, both studies suggested preoperative 3D image planning may help surgeons to decide optimal prosthesis size and position. In the current study, we also found that 3D templating allowed surgeons to predict the optimal size of component. When there is no proper size of implant, preoperative $3 \mathrm{D}$ planning may inspire surgeons to change the plan to optimize the fitness of the implant by changing the humeral neck cutting angle or position.

This 3D templating can be particularly useful for patients with a neck-shaft angle beyond normal range. In patients with a varus neck-shaft angle under $130^{\circ}$, an osteotomy can be made at the superolateral point in an inferomedial direction, avoiding the injury of greater tuberosity or rotator cuff while saving the medial aspect of humeral head. In case of an excessive valgus neck-shaft angle over $140^{\circ}$, the superolateral portion of humeral head should be remained during osteotomy for anatomical reduction. Several studies already discussed the biomechanical and clinical results related to anatomical reduction after total shoulder arthroplasty, ${ }^{13)}$ and several revised prostheses have been launched recently to facilitate anatomical reconstruction. These state-of-the-art prostheses allow for manipulation of the rotation and neck-shaft angle in response to anatomical variations of humeri. However, in some countries, these improved prostheses are not available, so surgeons have no choice but to use the fixed neck-shaft angle prostheses, which highlights the importance of accurate preoperative planning. In this case, 3D templating may be of significant assistance for anatomical reduction in total shoulder arthroplasty.

One of several limitations of this study is the small number of enrolled cases. In addition, the preoperative and postoperative radiographs used for evaluating anatomical parameters were not in the exact same plane, and this may have also caused some errors. Most contralateral humeri showed osteoarthritic changes, and this could have caused some limitation in measuring and comparing anatomical parameters. The computer program used for this study is not open to public yet, which makes it difficult to apply in general. Finally, we did not evaluate the relevance of this radiographic results to clinical outcome and to those in other studies.

In total shoulder arthroplasty, 3D templating seems to enable accurate prediction of sizes of prostheses to be inserted and replication of normal anatomy. Therefore, 3D templating and simulation program can be useful to avoid errors in reconstruction of normal anatomy of the shoulder during prosthetic joint replacement.

\section{CONFLICT OF INTEREST}

No potential conflict of interest relevant to this article was reported.

\section{REFERENCES}

1. Harryman DT, Sidles JA, Harris SL, Lippitt SB, Matsen FA 3rd. The effect of articular conformity and the size of the humeral head component on laxity and motion after glenohumeral arthroplasty: a study in cadavera. J Bone Joint Surg Am. 1995;77(4):555-63.

2. Williams GR Jr, Wong KL, Pepe MD, et al. The effect of articular malposition after total shoulder arthroplasty on glenohumeral translations, range of motion, and subacromial impingement. J Shoulder Elbow Surg. 2001;10(5):399-409.

3. Terrier A, Ramondetti S, Merlini F, Pioletti DD, Farron A. Biomechanical consequences of humeral component malpositioning after anatomical total shoulder arthroplasty. J Shoulder Elbow Surg. 2010;19(8):1184-90.

4. Favre P, Moor B, Snedeker JG, Gerber C. Influence of component positioning on impingement in conventional total shoulder arthroplasty. Clin Biomech (Bristol, Avon).
2008;23(2):175-83.

5. Pearl ML. Proximal humeral anatomy in shoulder arthroplasty: implications for prosthetic design and surgical technique. J Shoulder Elbow Surg. 2005;14(1 Suppl S):99S-104S.

6. Kwon YW, Powell KA, Yum JK, Brems JJ, Iannotti JP. Use of three-dimensional computed tomography for the analysis of the glenoid anatomy. J Shoulder Elbow Surg. 2005;14(1):8590.

7. Codsi MJ, Bennetts C, Gordiev K, et al. Normal glenoid vault anatomy and validation of a novel glenoid implant shape. J Shoulder Elbow Surg. 2008;17(3):471-8.

8. Iannotti JP, Gabriel JP, Schneck SL, Evans BG, Misra S. The normal glenohumeral relationships: an anatomical study of one hundred and forty shoulders. J Bone Joint Surg Am. 1992;74(4):491-500. 
Cho et al. Three-Dimensional Templating for Total Shoulder Joint Arthroplasty

Clinics in Orthopedic Surgery • Vol. 12, No. 2, $2020 \bullet$ www.ecios.org

9. Jeong J, Bryan J, Iannotti JP. Effect of a variable prosthetic neck-shaft angle and the surgical technique on replication of normal humeral anatomy. J Bone Joint Surg Am. 2009;91(8):1932-41.

10. Kilic M, End S, Irlenbusch U. Replication of the rotational center of the humeral head with second-generation stemmed prostheses. Acta Orthop Traumatol Turc. 2011;45(2):71-8.

11. Saifi C, Noticewala MN, Greiwe RM, et al. Reliability of 3-dimensional glenoid component templating and correla- tion to intraoperative component selection. Am J Orthop (Belle Mead NJ). 2017;46(5):E280-92.

12. Scalise JJ, Codsi MJ, Bryan J, Brems JJ, Iannotti JP. The influence of three-dimensional computed tomography images of the shoulder in preoperative planning for total shoulder arthroplasty. J Bone Joint Surg Am. 2008;90(11):2438-45.

13. Merolla G, Di Pietto F, Romano S, Paladini P, Campi F, Porcellini G. Radiographic analysis of shoulder anatomical arthroplasty. Eur J Radiol. 2008;68(1):159-69. 